\title{
Quantitative Atomic 3D Imaging of Single/Double Sheet Graphene Structure by Exit-Wave Reconstruction using a Titan G2 at $80 \mathrm{kV}$ Acceleration Voltage
}

\author{
J.R. Jinschek*, E. Yucelen***, H.A.Calderon***, B. Freitag* \\ * FEI Europe nanoPort, Eindhoven, Building AAE, Achtseweg Noord 5, P.O. Box 80066, 5600 \\ KA Eindhoven, The Netherlands \\ ** National Centre for HREM, Delft University of Technology, Delft, The Netherlands \\ *** ESFM-IPN, UPALM Zacatenco D. F. Mexico
}

New manmade Carbon-based nanostructures are considered to be essential new building blocks for bottom-up nanotechnology. For example, graphene arouse already great research interest [1] especially as it was found to exist with extraordinary electronic structure [2]. This extraordinary behavior of electrons is directly related to the crystal structure and the arrangement of every individual Carbon atom in this single sheet in 2-D, as well as, in case of few-layer graphene (FLG) in 3-D. Comparison between theoretical models as well as their predictions and the atomic positions - revealed by atomic-resolution experiment at the same length scale - is therefore a fundamental request before applications in nanoscale science and technology can be defined.

Drawbacks of imaging nanostructures using accelerated electrons are ionization damage by exciting electronic states and knock-on damage caused by collisions of beam electrons with specimen atoms. In graphene, the ionization damage is considered to be less important due to its high conductivity. The knock-on damage can be minimized by operating state-of-the-art electron microscopes at acceleration voltages below the material's knock-on damage threshold - such as in our case at $80 \mathrm{kV}$. The knock-on damage threshold for Carbon atoms in perfect graphene structure is estimated to be $113 \mathrm{kV}[3]$.

As another advantage of using an accelerating voltage of the electrons of $80 \mathrm{kV}$, the electron scattering becomes stronger at lower acceleration voltages. A drop in voltage to $80 \mathrm{kV}$ from $300 \mathrm{kV}$ improves the 'signal' of Carbon atoms (Fig.1a), allows for better S/N ratios, and therefore better sensitivity to detect small phase shifts carrying the 3-D structural information. At these low voltages usage of a spherical aberration $\left(C_{S}\right)$ corrector is essential to reduce imaging artifacts caused by aberrations due to unavoidable imperfections of the spherical objective lens, and, in this way, is essential to increase the achievable interpretable resolution and sensitivity to the point that atomicscale structures and single atom columns can be resolved.

With those settings the focal spread (more specific: the energy spread) is now limiting the achievable information limit in a non-chromatic-aberration corrected microscope [4] at a spatial resolution above the $\mathrm{C}-\mathrm{C}$ bond length in graphene of $1.42 \AA$. In the "rainbow illumination" approach [5], the XFEG gun and monochromator setting leads to a lower energy spread of about $0.2 \mathrm{eV}$, and therefore the tuned temporal coherence damping envelope of the phase contrast transfer function (CTF) allows an achievable spatial resolution down to at least $1.1 \AA$ (Fig.1b).

Exit-wave reconstruction has been performed using a focal series of HRTEM images of a graphene layer structure with lower dose image conditions (dose rate of $\sim 1.1 \cdot 10^{5} \mathrm{e}^{-} / \mathrm{s} \AA^{2}$ ). Here, we compare experimental data with simulations, and thereby demonstrate "seeing" single Carbon atoms in 3-D on single/double layer graphene (Fig.2). Graphene provides an ideal platform for demonstrating the potential of our achievement. The data presented can unambiguously distinguish between single and two atom columns in a double layer of graphene. Dopant of foreign atoms can be excluded by the 
linearity in the phase shift in the double atom positions. The sensitivity is even sufficient that a phase change caused by single Carbon atom positions can be obtained well above the noise level, which clearly quantitatively matches a position height change of $0.35 \mathrm{~nm}$ in double layers (Fig.2) [6].

\section{References}

[1] Geim, A. K., Science 324 (2009) 1530.

[2] A.H. Castro Neto et al., Reviews of Modern Physics 81 (2009) 109.

[3] A. Zobelli et al. Physical Review B 75 (2007) 245402.

[4] J. Barthel et al. Physical Review Letters 101 (2008) 200801.

[5] B. Freitag et al. Ultramicroscopy 102 (2005) 209.

[6] J.R. Jinschek et al. submitted (2010).

[7] The authors thank Ch. Kisielowski (NCEM \& Helios, LBNL, Berkeley, CA) for discussions. The sample is a courtesy of N. Alem \& A. Zettl (UC Berkeley, CA).
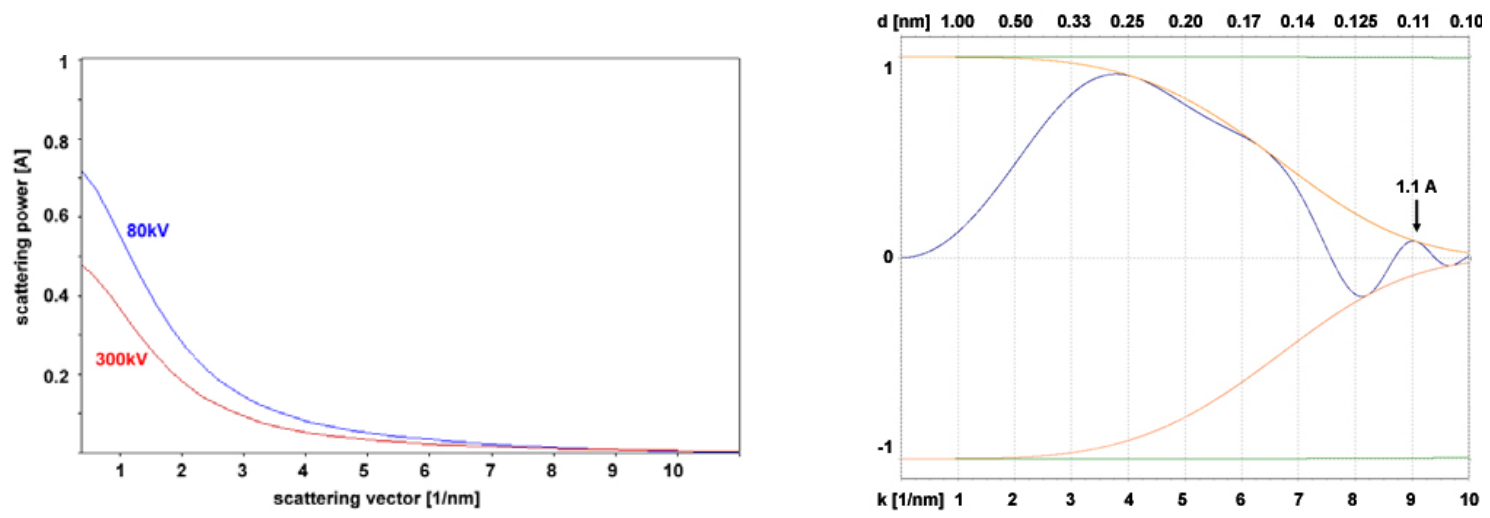

FIG. 1:

a) Electron scattering power of Carbon at $80 \mathrm{kV}$ (blue) vs. $300 \mathrm{kV}$ (red) (see Reimer)

b) Calculated phase contrast transfer function (blue curve) of the used microscope setting: $80 \mathrm{kV}$, defocus $=-7 \mathrm{~nm}, C_{S}=-0.015 \mathrm{~mm}, C_{C}=1.4 \mathrm{~mm}, \Delta \mathrm{E}=0.2 \mathrm{eV}$, focal spread $=2.07 \mathrm{~nm}$. Transfer of information at least up to a resolution of $1.1 \AA$ (see indication).
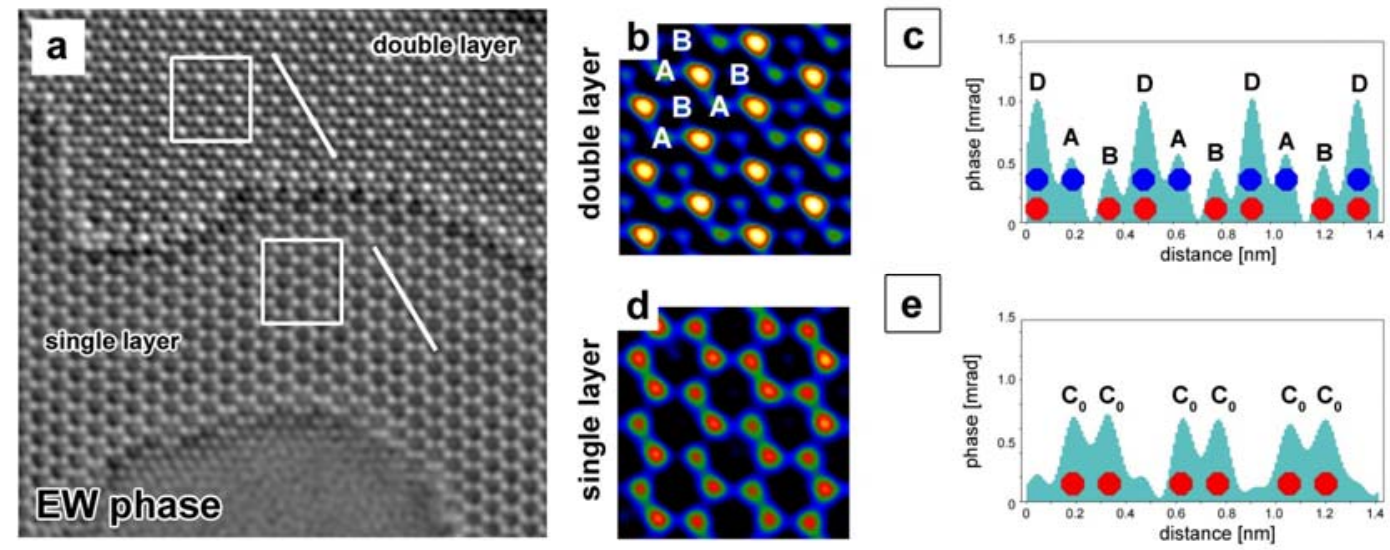

FIG. 2:

a) experimental EW phase image of graphene single/double layer structure

$b, d)$ higher magnification in RGB color scale: $b$ - from the double layer area, $d$ - from the single layer area, indicated by “ $\square$ ” in Fig. 2a

c, e) line scans of experimental phase image: $\mathrm{c}$ - from the double layer area, e - from the single layer area 